\title{
Stereotactic Robotic Body Radiotherapy for Patients With Unresectable Hepatic Oligorecurrence
}

\author{
Patrick Berkovic, Akos Gulyban, Paul Viet Nguyen, David Dechambre, \\ Philippe Martinive, Nicolas Jansen, Ferenc Lakosi, Levente Janvary, \\ Philippe A. Coucke
}

\section{Abstract}

\begin{abstract}
We present our retrospective study of $\mathbf{4 2}$ patients treated for hepatic oligorecurrence with stereotactic body radiotherapy using the CyberKnife system (Accuray Inc). Besides reporting on acute and late toxicities, the influence of patient and lesion characteristics on local control, liver and distant progression-free survival, and overall survival were also investigated.

Background: The purpose of this study was to analyze local control (LC), liver progression-free survival (PFS), and distant PFS (DFS), overall survival (OS), and toxicity in a cohort of patients treated with stereotactic body radiotherapy (SBRT) with fiducial tracking for oligorecurrent liver lesions; and to evaluate the potential influence of lesion size, systemic treatment, physical and biologically effective dose (BED), treatment calculation algorithms and other parameters on the obtained results. Patients and Methods: Unoperable patients with sufficient liver function had [18F]-fluorodeoxyglucose-positron emission tomography-computed tomography and liver magnetic resonance imaging to confirm the oligorecurrent nature of the disease and to further delineate the gross tumor volume (GTV). An intended dose of 45 Gy in 3 fractions was prescribed on the $80 \%$ isodose and adapted if risk-related. Treatment was executed with the CyberKnife system (Accuray Inc) platform using fiducials tracking. Initial plans were recalculated using the Monte Carlo algorithm. Patient and treatment data were processed using the Kaplan-Meier method and log rank test for survival analysis. Results: Between 2010 and 2015, 42 patients (55 lesions) were irradiated. The mean GTV and planning target volume (PTV) were $30.5 \mathrm{cc}$ and $96.8 \mathrm{cc}$, respectively. Treatments were delivered 3 times per week in a median of 3 fractions to a PTV median dose of 54.6 Gy. The mean GTV and PTV D98\% were 51.6 Gy and 51.2 Gy, respectively. Heterogeneity corrections did not influence dose parameters. After a median follow-up of 18.9 months, the 1- and 2-year LC/liver PFS/DFS/OS were 81.3\%/55\%/62.4\%/86.9\%, and 76.3\%/42.3\%/52\%/78.3\%, respectively. Performance status and histology had a significant effect on LC, whereas age (older than 65 years) marginally influenced liver PFS. Clinical target volume physical dose V45 Gy $>95 \%$, generalized equivalent uniform dose $(a=-30)>45$ Gy and a BED $(\alpha / \beta=10)$ V105 Gy $>96 \%$ showed statistically significant effect on the LC. Acute Grade 3 gastrointestinal (Gl) and late Grade 2 Gl and fatigue toxicity were found in $5 \%$ and $11 \%$ patients, respectively. Conclusion: Favorable survival and toxicity results support the potential paradigm shift in which the use of SBRT in oligorecurrent liver disease could benefit patients with unresectable or resectable liver metastases.
\end{abstract}

Clinical Colorectal Cancer, Vol. 16, No. 4, 349-57 (c) 2017 Elsevier Inc. All rights reserved.

Keywords: Liver metastasis, Oligometastasis, Outcome, Prognostic factors, SBRT

Department of Radiation Oncology, University Hospital of Liège, Liege, Belgium

Submitted: Aug 2, 2016; Revised: Jan 20, 2017; Accepted: Mar 9, 2017; Epub: Mar 21,2017

Address for correspondence: Patrick Berkovic, MD, Avenue de l'hôpital 1, Campus Sart Tilman B35, Liege, 4000, Belgium

E-mail contact: p.berkovic@gmail.com

\section{Introduction}

The most common metastatic lesion to the liver is from colorectal origin. ${ }^{1}$ Approximately $50 \%$ of colorectal cancer (CRC) patients will be diagnosed with either synchronous or metachronous liver metastasis. ${ }^{2,3}$ Without treatment, the 3-year survival rates remain dismal at $3 \%{ }^{4,5}$ The standard treatment for hepatic oligometastasis is surgical resection, ${ }^{6,7}$ with a 


\section{SBRT for Unresectable Hepatic Oligorecurrence}

potential curative intent and 5 -year survival rates of $25 \%$ to $50 \%$ and 10 -year overall (OS) up to $22 \%$ to $43 \%{ }^{8,9}$ Despite improved surgical techniques, ${ }^{10}$ only a small (approximately $25 \%)$ proportion of patients with hepatic oligometastases are eligible for surgical resection mainly because of medical or technical contraindications. ${ }^{11}$ Over the past years, other local treatment options have emerged such as embolization, ${ }^{12}$ thermic ablation, and radiotherapy. These techniques improve long-term tumor control and progression-free survival in case of inoperable disease, but data on OS benefit are scarce. ${ }^{13-19}$ Stereotactic body radiotherapy (SBRT) allows delivery of focal ablative doses to the hepatic metastases while sparing the normal hepatic tissue and surrounding organs at risk. ${ }^{20,21}$ Numerous studies have now confirmed the feasibility, low toxicity, and efficacy of such treatments. ${ }^{22,23}$ In case of limited metastatic disease (up to 5 metastatic lesions), local control (LC) varies between $62 \%$ and up to $92 \%$ and OS rates between $30 \%$ (2-year) and $72 \%$ (1-year) with low rates of Grade 3 toxicity. ${ }^{8,24-26}$ The challenge of SBRT to deliver high radiation doses to the liver is to manage respiratory movements. Various breathing control strategies such as respiratory gating and motion management techniques have been implemented to overcome this limitation, ${ }^{27}$ including the CyberKnife system (Accuray Inc), which performs real-time tumor tracking. This allows for a high level of precision while treating the patient in free breathing and still maintaining patient comfort. The primary aim of our retrospective study was to report on LC, liver and distant progression-free survival, and OS of patients treated for pure hepatic oligorecurrence (as defined by Niibe and Hayakawa ${ }^{28}$ ) with SBRT using the CyberKnife system, including the observed acute and late toxicity.

\section{Patients and Methods}

Between September 2010 and July 2015 consecutive patients with up to 3 synchronous liver metastases were included in this study for CyberKnife treatment at the Liege University Hospital. All patients were referred for stereotactic treatment after a full staging including baseline registration of the liver function, chest and abdominal diagnostic computed tomography (CT) and hepatic magnetic resonance imaging (MRI) or [18F]-fluorodeoxyglucose (FDG) positron emission tomography (PET)-CT imaging, confirming the absence of tumoral activity at the primary tumor site and extrahepatic metastases. Patients were informed of the intent, side effects, and practical modalities of the treatment and consent for treatment was obtained before SBRT. All patients were considered unsuitable candidates for surgical resection. We included lesions in any location within the liver, irrespective of previous treatments including chemotherapy, surgery, or other local treatments such as previous radiotherapy for metachronous hepatic metastases. Patients with heavily compromised liver function tests (ie, Child-Pugh class C, platelet count $<60 \times 109 / \mathrm{L}$ and hemoglobin $<8 \mathrm{~g} / \mathrm{dL}$, and/or liver enzymes $>3$ times the upper limit of normal), ascites, tumors $>6 \mathrm{~cm}$ in diameter, uncontrolled primary tumor, and patients with an expected life expectancy of $<6$ months were excluded from standard SBRT treatment. Grade 3 toxicity or Radiation Induced Liver Disease (RILD) were relative exclusion criteria for reirradiation.

\section{CyberKnife Planning and Treatment}

All patients had radio-opaque 3-mm long gold fiducials (Goldlock; Beampoint) placed using transabdominal puncture with CT guidance by a dedicated interventional radiologist. The 3 intended fiducials were placed according to Accuray guidelines. The treatment simulation took place at least 1 week later to avoid marker migration between the simulation and the start of the treatment. Patients were positioned in the supine position in a vacuum bag (Orfit), using a knee and feet support system with arms next to the body. A multislice CT scan was obtained with a slice thickness of $1 \mathrm{~mm}$ using a CT simulator (Brilliance BigBore CT scan, Philips). After the first scan series in mild expiration, a second scan was obtained after intravenous iodine-based contrast injection. Additional PET-CT imaging was obtained in the treatment position, using the same immobilization devices followed by liver MRI, without the vacuum bag. All Digital Imaging and Communications in Medicine image series were rigidly coregistered using the Multiplan treatment planning system (Accuray, Inc) with special attention with regard to proper matching on the fiducials. During treatment planning the following organs at risk were delineated: left and right lung, esophagus, heart, thoracic wall or ribs, left and right kidneys, intestinal structures, stomach, spinal canal, whole liver, great vessels, and a 4-mm skin area.

A gross tumor volume (GTV) was defined on the expiration reference CT scan, using mutual information from all available fused images. A clinical target volume (CTV) margin of $5 \mathrm{~mm}$ was added and manually corrected in function of liver capsule and adjacent structures. A uniform 3-mm margin was used to create the planning target volume (PTV). For most of the patients, the intended prescription dose was 45 Gy in 3 fractions to the $80 \%$ isodose line. Aimed PTV coverage was $95 \%$ with the prescription dose. In case of reirradiation, former treatment plans were taken into account by either using the previous isodose lines for the optimization purpose (before December 2014) or proper physical dose summation of irradiation(s) with the actual plan (after December 2014) using Mirada RTx (version 1.6.2/1.6.2, Mirada Medical). The organs at risk dose constraints reported by Timmerman ${ }^{29}$ were always respected, at cost of altered fractionation and if required, sacrificing the target coverage. The estimated fraction duration was kept in all cases $<70$ minutes. The treatment was delivered using a CyberKnife VSI system with Robocouch and planned using Multiplan treatment planning software with Raytracing algorithm (version 4.6, Accuray). The treatment plan was retrospectively recalculated using a Monte Carlo (MC) dose calculation algorithm (version 5.1), to evaluate the potential effect on the dose distribution of using equivalent path length heterogeneity correction algorithm in the lung vicinity. Synchrony Respiratory Tracking System was used to continuously track fiducial position and synchronize beam delivery with respiratory motion. Tracking was ideally performed on all available fiducials but was adapted in case of suboptimal fiducial visibility. The treatment duration was recorded and the treatment sessions were each delivered at an interval of a minimum of 40 hours. Patients were prescribed proton pump inhibitors, serotonin 5-hydroxytryptamin antagonists, benzodiazepines, and antacids in function of the symptomatology. 


\section{Follow-up and Toxicity Evaluation}

Patients were evaluated after the last fraction, at 2 weeks, and then in function of the referring team at an interval of 2 to 4 months. At each outpatient contact a clinical examination and the treatment toxicity was assessed. Treatment response was evaluated using serial contrast enhanced spiral CT or MRI scans and was defined according to the Response Evaluation Criteria in Solid Tumors version 1.1. ${ }^{30} \mathrm{LC}$ was defined as complete response (CR), partial response $(\mathrm{PR})$, or stable disease. Additional metachronous liver lesions were scored as liver-specific progression. Toxicity was evaluated using the Common Terminology Criteria for Adverse Events version 4.0. ${ }^{31}$ Toxicities occurring $\leq 3$ months after SBRT were considered acute whereas toxicities occurring after 3 months were considered late.

\section{Data Analysis}

Local control, liver progression free-survival (PFS), distant PFS (DFS), and overall survival (OS) curves were evaluated using the Kaplan-Meier method. All data for LC were determined for each lesion separately, whereas the liver PFS, DFS, and OS per patient originated from the day of the first SBRT treatment. Survivalrelated subgroup analysis was performed using the log rank test. For LC, additional investigation included treatment planningrelated parameters such as number of fractions, target volumes, physical, and biological effective dose (BED; BED 10 considering $\alpha / \beta=10)$ coverage and generalized equivalent uniform dose (gEUD, a $=-30$ ) for CTV and PTV in a systematic manner. For all tests, a $P$ value $<.05$ was considered as statistically significant using Python packages (Pandas version 0.15.2, Scipy version 0.14.0, and Lifelines version 0.9.0.0). ${ }^{32}$

\section{Results}

During the period from September 2010 to September 2015, a total of 42 patients with 55 lesions were irradiated in 48 treatments. Eight and 2 patients were treated for, respectively, 2 and 3 synchronous lesions. Pretreatment PET-CT and MRI was available for all patients. A median of 3 (range, 2-5) fiducials was inserted. Further population and tumor characteristics are presented in Table 1.

\section{Treatment Characteristics}

The mean GTV and PTV were $30.5 \mathrm{cc}$ (SD, 26.3) and $96.8 \mathrm{cc}$ (SD, 59.6), respectively. All treatments were delivered 3 times per week in a median of 3 fractions (range, 3-6) to an average PTV median dose of 54.6 Gy (range, 29.1-58.9). This corresponds to an average median PTV BED 10 of 132 Gy (range, 57.5-174.76). The mean GTV and PTV D98\% were 51.6 Gy (SD, 6.2) and 51.2 Gy (SD, 6.6), respectively. Dosimetric parameters are presented in Table 2. Each treatment was delivered by an average of 137 (range, 63-224) beams and had an average duration of 41 minutes (range, 24-68 minutes). All dose constraint parameters reported by Timmerman were respected and the dose to $700 \mathrm{cc}$ of uninvolved liver tissue was consistently kept at $<15$ Gy. Retrospective recalculation with the MC algorithm showed a deviation of $-0.3 \%$ mean dose for target volumes (range, $-1.7 \%$ to $2.1 \%$ ), whereas for all organs at risk was $-1.7 \%$ (range, $-7.5 \%$ to $2.2 \%$ ) compared with the Raytracing algorithm. These differences were considered

\begin{tabular}{|c|c|c|}
\hline Characteristic & $\begin{array}{l}\text { Per Patient (First } \\
\text { Lesion Counts) }\end{array}$ & $\begin{array}{c}\text { Per Lesion } \\
\text { Per Treatment }\end{array}$ \\
\hline \multicolumn{3}{|l|}{ Sex, n (\%) } \\
\hline Female & $19(45.2)$ & $25(46.3)$ \\
\hline Male & $23(54.8)$ & $29(53.7)$ \\
\hline $\begin{array}{l}\text { Median Age at SBRT } \\
\text { (Range), y }\end{array}$ & 67.1 (43.1-83.3) & $68.4(43.1-83.3)$ \\
\hline $\begin{array}{l}\text { Median Age at Diagnosis } \\
\text { (Range), y }\end{array}$ & $66.9(42.5-83)$ & $67.9(42.5-83)$ \\
\hline \multicolumn{3}{|l|}{ Primary Site, n (\%) } \\
\hline Colorectal & 25 (59.5) & 30 (55.6) \\
\hline Breast & $7(16.7)$ & $11(20.4)$ \\
\hline Other & $5(11.9)$ & $6(11.1)$ \\
\hline Lung & $3(7.1)$ & $3(5.6)$ \\
\hline Stomach & $1(2.4)$ & $2(3.7)$ \\
\hline Unknown & $1(2.4)$ & $1(1.9)$ \\
\hline Melanoma & $1(2.4)$ & $1(1.9)$ \\
\hline \multicolumn{3}{|l|}{$\begin{array}{l}\text { Lesion Treated at the Same } \\
\text { Time (on the Same } \\
\text { Computed Tomography } \\
\text { Scan), n (\%) }\end{array}$} \\
\hline 1 & 32 (76.2) & - \\
\hline 2 & $8(19)$ & - \\
\hline 3 & $2(4.8)$ & - \\
\hline \multicolumn{3}{|l|}{$\begin{array}{l}\text { Performance Status, } \\
\text { n (\%) }\end{array}$} \\
\hline 0 & $20(47.6)$ & $25(46.3)$ \\
\hline 1 & $22(52.4)$ & $29(53.7)$ \\
\hline \multicolumn{3}{|l|}{$\begin{array}{l}\text { Previous Local Treatment, } \\
\text { n (\%) }\end{array}$} \\
\hline No & $26(61.9)$ & $31(57.4)$ \\
\hline Surgery & $11(26.2)$ & $15(27.8)$ \\
\hline Radiofrequency & $10(23.8)$ & 12 (22.2) \\
\hline Radiotherapy & $5(11.9)$ & $5(9.3)$ \\
\hline \multicolumn{3}{|l|}{$\begin{array}{l}\text { Previous Chemotherapy, } \\
\text { n (\%) }\end{array}$} \\
\hline Yes & 35 (83.3) & 43 (79.6) \\
\hline No & $7(16.7)$ & $11(20.4)$ \\
\hline
\end{tabular}

clinically irrelevant using MC either with fiducial-induced artifacts (native CT) or with water equivalent liver (density overwrite) leading to a target mean dose difference $<1 \%$.

\section{Treatment Efficacy}

A post-treatment evaluation scan was available for all of the treated lesions. Of the 55 treated lesions, 22 lesions (40.7\%) were defined as a CR, $14(25.9 \%)$ as a PR, and $8(14.8 \%)$ lesions were stable, whereas 10 lesions (18.5\%) progressed. A typical CR on the basis of before and follow-up FDG PET/CT is presented in Figure 1. After a median follow-up of 18.9 months (range, 3.250.4), the 1 - and 2 -year LC was $81.3 \%$ and $76.3 \%$, respectively, which remained stable thereafter. The corresponding liver PFS and DFS rates were $55.0 \%$ and $42.3 \%$, and $62.4 \%$ and $52.0 \%$, respectively. Associated OS rates were $86.9 \%$ and $78.3 \%$, respectively (Figure 2). Performance status (0 vs. $1+)$ and histology 
Table 2 Treatment-related Parameters

\begin{tabular}{|c|c|}
\hline Parameter & Value \\
\hline \multicolumn{2}{|l|}{ Fractions, n (\%) } \\
\hline 3 & $39(72.2)$ \\
\hline 4 & $1(1.9)$ \\
\hline 5 & $12(22.2)$ \\
\hline 6 & $2(3.7)$ \\
\hline \multicolumn{2}{|l|}{ RECIST, n (\%) } \\
\hline Complete remission & $22(40.7)$ \\
\hline Partial remission & $14(25.9)$ \\
\hline Stable disease & $8(14.8)$ \\
\hline Progressive disease & $10(18.5)$ \\
\hline \multicolumn{2}{|l|}{ Mean Volume (SD) } \\
\hline Gross tumor volume & $30.6(26.3)$ \\
\hline Clinical target volume & $67.6(48.2)$ \\
\hline Planning target volume & $96.9(59.6)$ \\
\hline \multicolumn{2}{|c|}{ Mean Treatment Executions (SD) } \\
\hline Beams, $n$ & $137(38)$ \\
\hline Treatment time, min & $44.1(10.9)$ \\
\hline \multicolumn{2}{|c|}{ Mean OAR Dose Constraints (SD) } \\
\hline \multicolumn{2}{|l|}{ Spinal cord } \\
\hline D $1.0 \mathrm{~mL}$ & $5.8(3.1)$ \\
\hline D $0.25 \mathrm{~mL}$ & $6.3(3.3)$ \\
\hline $\mathrm{D} \max$ & $6.9(3.6)$ \\
\hline \multicolumn{2}{|l|}{ Esophagus } \\
\hline D $5.0 \mathrm{~mL}$ & $3.7(3.8)$ \\
\hline Dmax & $7.8(7)$ \\
\hline \multicolumn{2}{|l|}{ Heart } \\
\hline D $15.0 \mathrm{~mL}$ & $7.2(6.1)$ \\
\hline Dmax & $13.5(10.2)$ \\
\hline \multicolumn{2}{|l|}{ Great vessels } \\
\hline D $10.0 \mathrm{~mL}$ & $8.6(6.2)$ \\
\hline Dmax & $16.3(11.1)$ \\
\hline \multicolumn{2}{|l|}{ Stomach } \\
\hline D $10.0 \mathrm{~mL}$ & $8.9(4.7)$ \\
\hline Dmax & $14.3(7.5)$ \\
\hline \multicolumn{2}{|l|}{ Duodenum } \\
\hline D $5.0 \mathrm{~mL}$ & $13(5.2)$ \\
\hline Dmax & 21.1 (9.3) \\
\hline \multicolumn{2}{|l|}{ Lungs } \\
\hline D $1500.0 \mathrm{~mL}$ & $1.6(0.9)$ \\
\hline D $1000.0 \mathrm{~mL}$ & $2.6(1.4)$ \\
\hline \multicolumn{2}{|l|}{ Liver } \\
\hline D $700.0 \mathrm{~mL}$ & $7.8(5.6)$ \\
\hline \multicolumn{2}{|l|}{ Renal cortex } \\
\hline D $200.0 \mathrm{~mL}$ & $3.1(2.6)$ \\
\hline
\end{tabular}

Abbreviations: $D$ " $X "=$ dose to the corresponding " $X$ " volume of a given organ; Dmax = maximum dose of the organ; OAR = organs at risk; RECIST $=$ Response Evaluation Criteria In Solid Tumors.

(adenocarcinoma vs. other) showed a statistically significant effect on LC (Figure 3). All other parameters did not appear to change significantly time-related outcomes (Table 3; Supplemental Figure 1 in the online version). Target volumes and number of fractions did not significantly change LC. For CTV, a physical dose coverage of $45 \mathrm{~Gy}$ to at least $95 \%$ of the volume, at least a $45 \mathrm{~Gy}$ gEUD $(\mathrm{a}=-30)$ and a BED coverage $(\alpha / \beta=10)$ of $105 \mathrm{~Gy}$ to at least $96 \%$ of the volume showed a statistically significant effect on LC. Similar to PTV, were $95 \%$ volume coverage $>43$ Gy, 40 Gy gEUD $(\mathrm{a}=-30)$, and $98 \%>85$ Gy BED 10 considered a critical threshold for the outcome measures.

\section{Toxicity}

No toxicities were observed during or after fiducial placement. The SBRT treatment was well tolerated with observed acute toxicities ranging from Grade 1 to 2 with an absolute rate of acute Grade 3 gastrointestinal (GI) toxicity in 3 treatments (5\%) including 1 case of RILD. Late toxicities also remained mild with $6(11 \%)$ observed Grade 2 GI $(n=4)$ and fatigue $(n=2)$. One patient was hospitalized for investigation and intravenous treatment because of GI toxicities (colic) and 1 patient died 10 months after SBRT after GI hemorrhage. The treatment plan of this patient was extensively analyzed and all treatment parameters and constrains were well below tolerance. The patient had various comorbidities including type 2 diabetes, Grade 2 esophagitis, hypertension, and was treated with aspirin for a previous cardiac event.

\section{Discussion}

Liver metastasis from solid tumors, particularly from primary CRC, represent an important clinical challenge. If left untreated the prognosis is poor with a median survival of $<1$ year. $^{9}$ In case of multiple liver metastases, chemotherapy remains the mainstay of the treatment. ${ }^{7}$ However, if oligometastatic disease is diagnosed, metastasis-directed local treatments might be considered. ${ }^{8,25}$ Numerous studies have shown the efficacy and survival benefit of surgical resection with a median survival between 40 and 64 months.' Unfortunately, most patients are found to be either surgically or medically unoperable. In recent years, radiotherapy has emerged as an effective and well tolerated treatment for patients with liver metastasis. Using modern stereotactic technology, including MRI and PET/CT imaging for accurate tumor delineation, intensity modulated radiotherapy and image-guided radiotherapy technology including tumor tracking for accurate treatment delivery, it has become the current standard in unresectable liver metastases treatment. ${ }^{22}$

\section{Local Control}

We report on 42 patients with 55 liver metastases, mostly from primary CRC, treated with a CyberKnife system with a median follow-up of 18.9 months. Our 1- and 2-year LC rates of approximately $80 \%$ are comparable with previously published data describing a cohort of liver metastasis only.' In fact, numerous studies have consistently shown the deleterious effect of previous chemotherapy, probably because of possible selection of radioresistant cells, and worst LC rates of liver metastases from colorectal origin. Our study concurs with the latter data in a subgroup analysis, besides performance status, colorectal adenocarcinoma had a statistically significant worst LC than other histologies. ${ }^{1,33}$ However, we could not confirm a significant effect on LC in our heavily 
Figure 1 Pretreatment Fused Positron Emission Tomography (PET)-Computed Tomography (CT) (Lefi) Shows the CyberKnife Highly Conformal Dose Distribution Targeted at the PET-Hypermetabolism Region, Gross Tumor Volume (Red), Using Fiducials (Black Arrow) Tracking. The 11-month Post-treatment (Right) PET-CT Shows No Hyperactivity Within the Liver, Indicating a Complete Remission

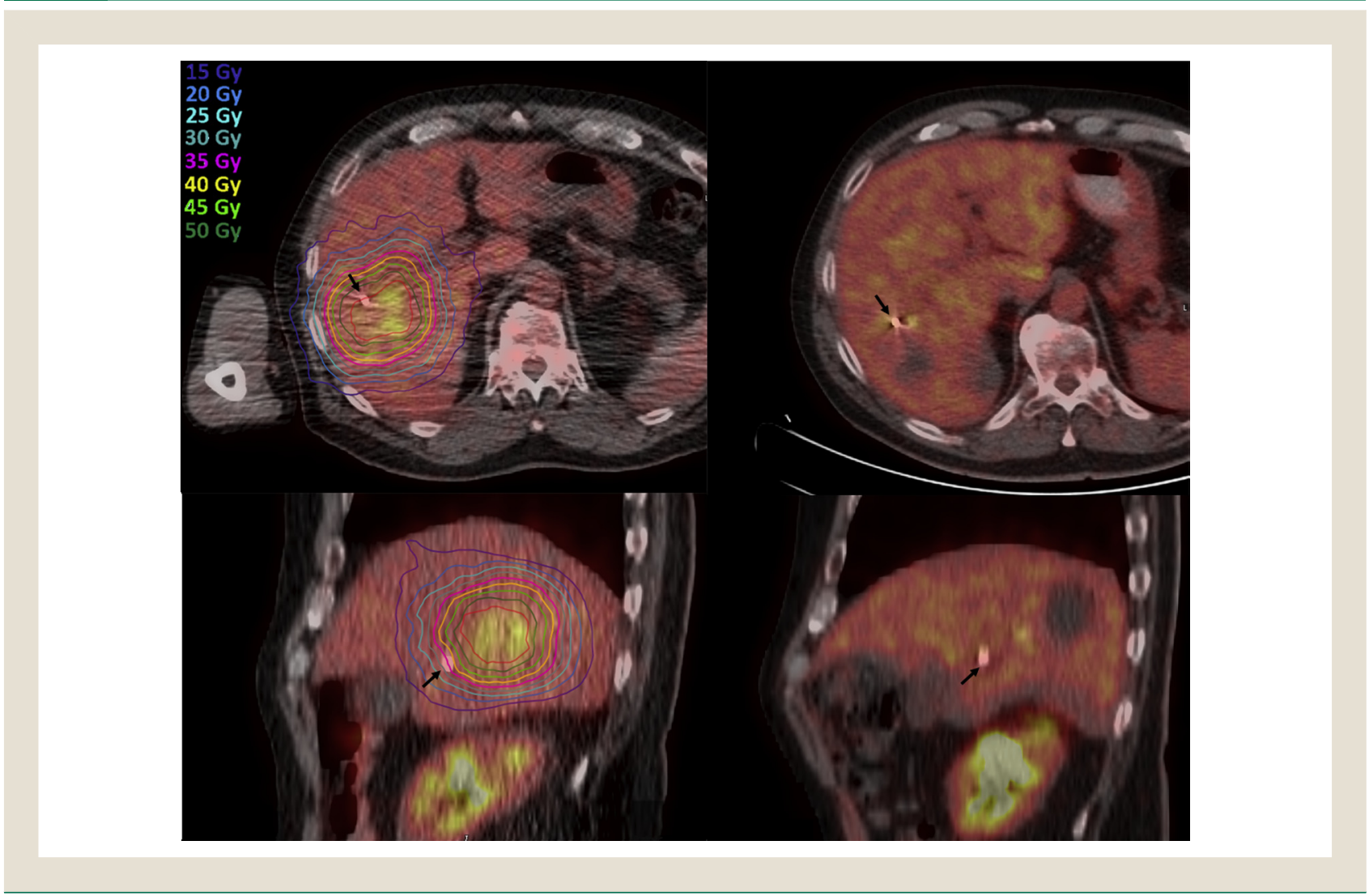

pretreated cohort in which, in fact, $80 \%$ received chemotherapy before SBRT. Moreover, in contrast to previous publications, we could not find any significant correlation between GTV size and LC. ${ }^{34}$ Despite the extensive use of SBRT in the oligometastatic setting, the precise radiobiology and cell-killing effects of this hypofractionated schedules have not been fully understood. Numerous studies have shown a dose response for LC but there is still uncertainty on the optimal threshold dose. We addressed this particular issue and suggest a prescription threshold dose of $45 \mathrm{~Gy}$ on the CTV and $>43$ Gy on the PTV to at least $95 \%$ of both volumes to significantly improve LC. This dose is on the lower end of the recommended prescription dose of $48 \mathrm{~Gy}$ in 3 fractions described in the review report by Høyer et al. ${ }^{22}$ However, this recommendation is on the basis of heterogeneous studies with very different tumor histologies and fraction sizes ranging from 1 to 6 fractions. In a pooled analysis by Chang et $\mathrm{al},{ }^{34}$ a similar dose response is observed with a required prescription dose of 46 to 52 Gy in 3 fractions to achieve a $90 \%$ LC. Rusthoven et $\mathrm{al}^{26}$ observed LC rates $>90 \%$ with a fractionation schedule of 3 fractions of 20 Gy. In contrast, Takeda et $\mathrm{al}^{35}$ improved the $\mathrm{LC}$ rates by increasing the dose to the lesion up to 83 till 100 Gy maximum dose with the prescribed dose of 50 to $60 \mathrm{~Gy}$ in 5 fractions on the $60 \%$ isodose to encompass the PTV. By using BED to account for different dose fractionation schedules, we observed a significant effect on LC for a
BED of 105 Gy to at least $95 \%$ of the volume, again, lower than the 117 Gy proposed by Chang et $\mathrm{al}^{34}$ to achieve $90 \%$ LC.

\section{Liver PFS}

The only factor shown to marginally correlate with liver PFS was the age of the patient (older or younger than 65 years, with $P=.07$ ). In our experience the 1 - and 2 -year liver PFS was $55 \%$ and $42.3 \%$, respectively. Our high rates, compared with the literature, are despite that our population was heavily pretreated. ${ }^{\text {? }}$

\section{Overall Survival}

Despite the selection bias of our patient population with most patients classified according to the World Health Organization as 1 with several comorbidities, who were considered clinically inoperable, our 1 - and 2 -year OS rates were $86.9 \%$ and $78.3 \%$, respectively. These numbers are at the higher end compared with the recent SBRT literature with 1-year OS rates between $67 \%$ and $85 \%{ }^{8,24,25}$ Compared with the surgical resection series and tumor ablation series our data are also at the higher end with 1-year OS rates in the range between $71 \%$ and $93 \%$ for surgery and $71 \%$ to $88 \%$ for tumor ablation. ${ }^{11}$ Although LC rates have been shown in previous studies to be a key determinant of OS, our high OS rates are probably because of our rigid inclusion criteria. All of our patients were (PET-CT confirmed) exclusively oligorecurrent in the 
Figure 2 Kaplan-Meier Curves and Log-Rank Test for Local Control, Liver Progression-free Survival (PFS), Disease-free Survival, and Overall Survival for Patient-related Parameters
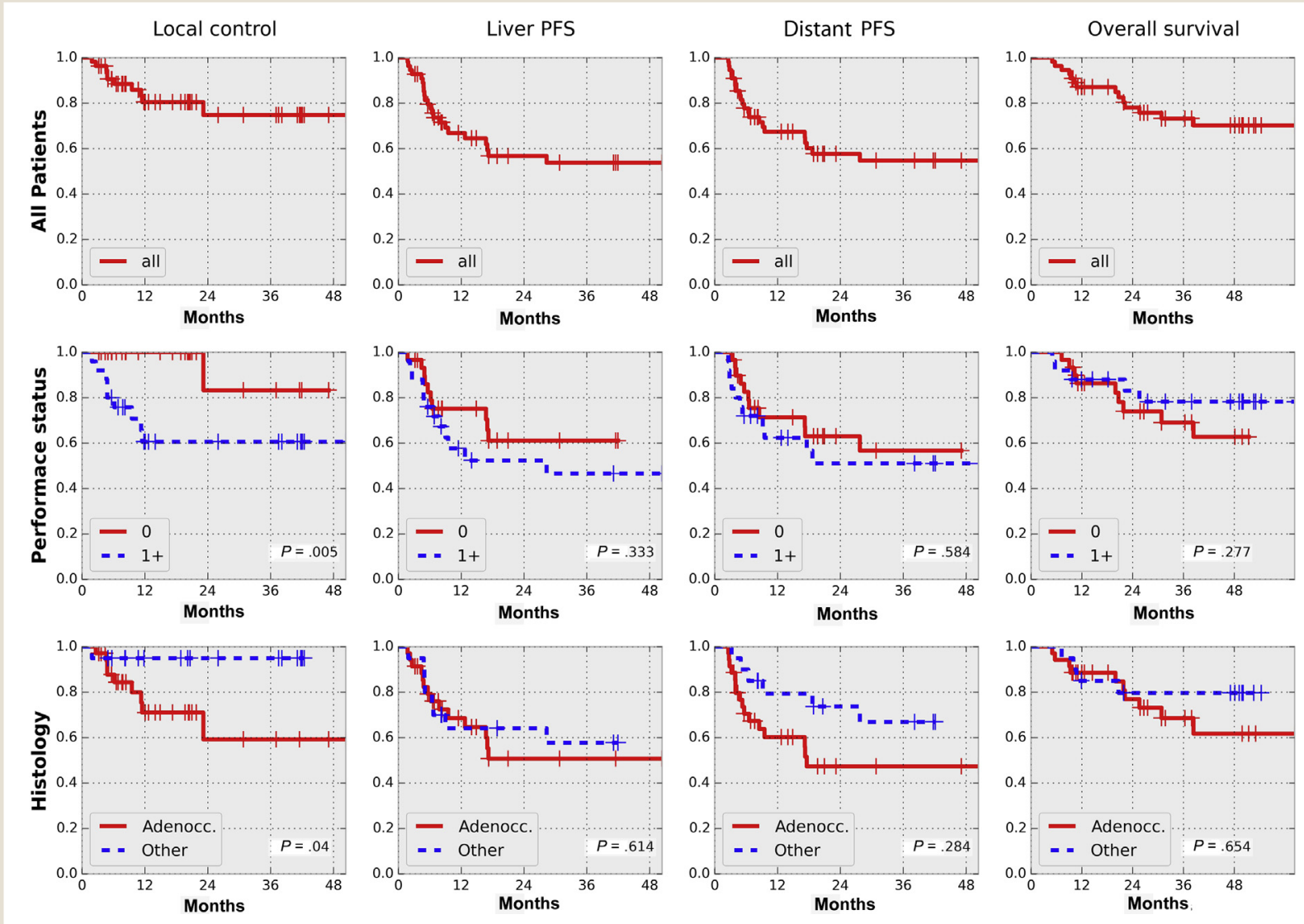

Abbreviation: Adenocc $=$ adenocarcinoma.

liver with the absence of tumoral activity at the primary site according to the concept proposed by Niibe and Hayakawa. ${ }^{28}$

\section{Toxicity}

Despite our relatively large PTVs (mean $=96.9 \mathrm{cc}, \mathrm{SD}=59.6 \mathrm{cc}$ ) we observed only 3 Grade 3 GI toxicities. One case of RILD was reported, which subsided after conservative treatment. One patient with late Grade 3 toxicities was observed and 1 patient died 10 month after SBRT treatment, probably because of his numerous comorbidities. None of our patients developed signs of liver failure or jaundice. These observations are in line with previously published low toxicity rates after CyberKnife treatment. ${ }^{1,36,37}$ Consistently respecting the liver constraint parameters whereby not less than $700 \mathrm{cc}$ of uninvolved liver tissue received more than 17.1 Gy in 3 fractions, along with the vacuum bag and the precision of the CyberKnife Synchrony tracking system, which allowed the delivery of high doses to the lesion while safeguarding the liver function. This allowed us to treat most of our patients (30 [72.2\%] out of 42) with the intended higher BED prescription of 3 fractions of $15 \mathrm{~Gy}$ compared with the other risk-adapted fractionation schemes. Furthermore, we observed no toxicity besides mild chest wall pains after fiducial placement.
Similar to Swaminath et al, ${ }^{38}$ we found a direct effect of the target volume coverage on the basis of the treatment planning parameters and LC, showing that the intended prescribed dose alone does not guarantee the effectiveness of the treatment. In their study patients were treated using Linac-based SBRT and four dimensional computer tomography approach and the accumulated GTV and minimum PTV dose was determined according to deformable dose accumulation (on the basis of 2-dimensional projections). Using CyberKnife with real-time tumor tracking, the physical, biological, and gEUD dose on the PTV and CTV with certain thresholds resulted as relevant. On the basis of these results, one might conclude that treatments for which sufficient target coverage cannot be achieved need to be reconsidered, because without reaching certain levels on dose delivery, the added value of local SBRT is not guaranteed for LC.

\section{Limitations}

Limitations of the current study include the retrospective nature and the mild variation in treatment schedules and delivered doses. The variability and quantity of previous chemotherapy schedules might also bias our LC and OS rates because of possible selection of radioresistant cells. ${ }^{39}$ Furthermore, no control group was available to 

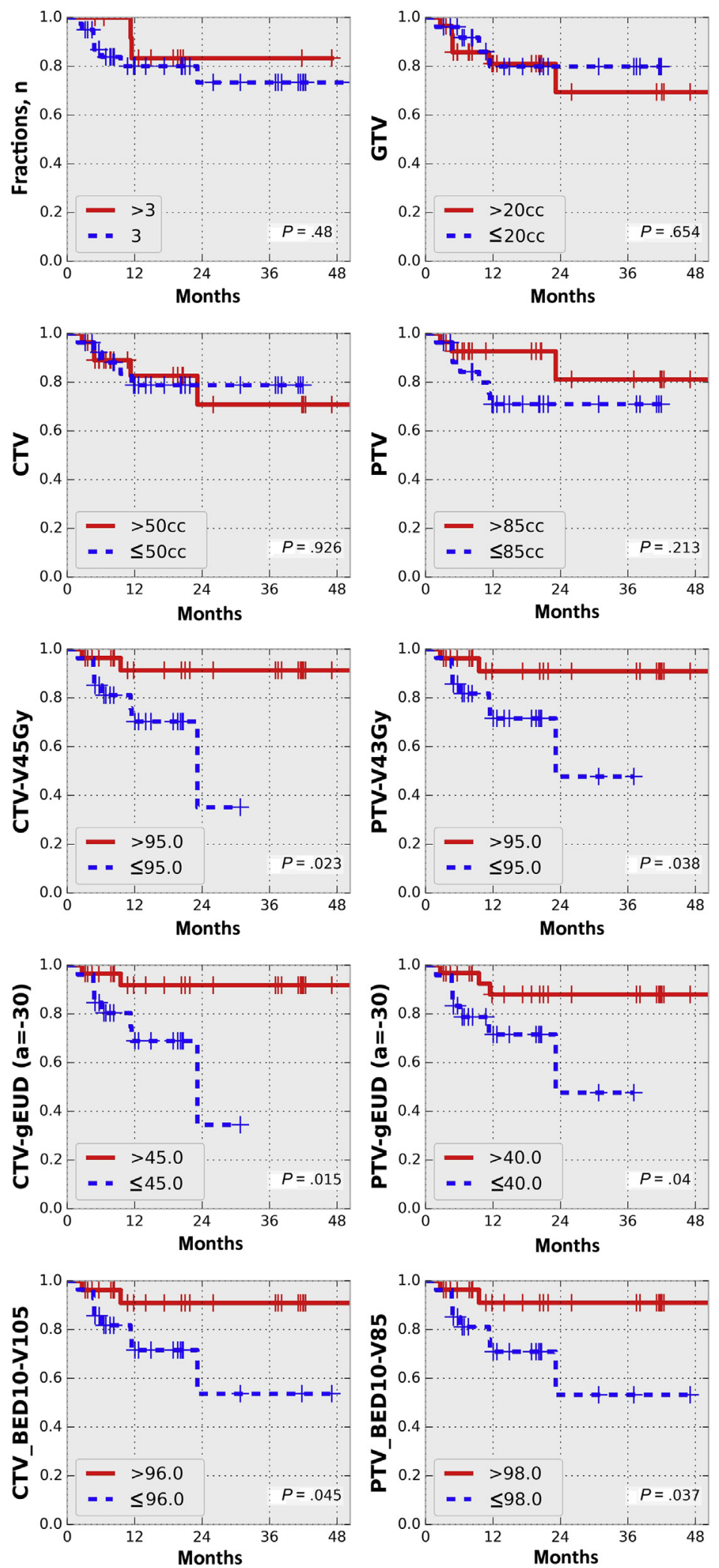

Abbreviations: BED = biological effective dose; CTV = clinical target volume; gEUD = generalized equivalent uniform dose; GTV = gross tumor volume; PTV = planning target volume; V“ $\mathrm{X}$ " = volume covered by the dose of " $X$ " Gy. 


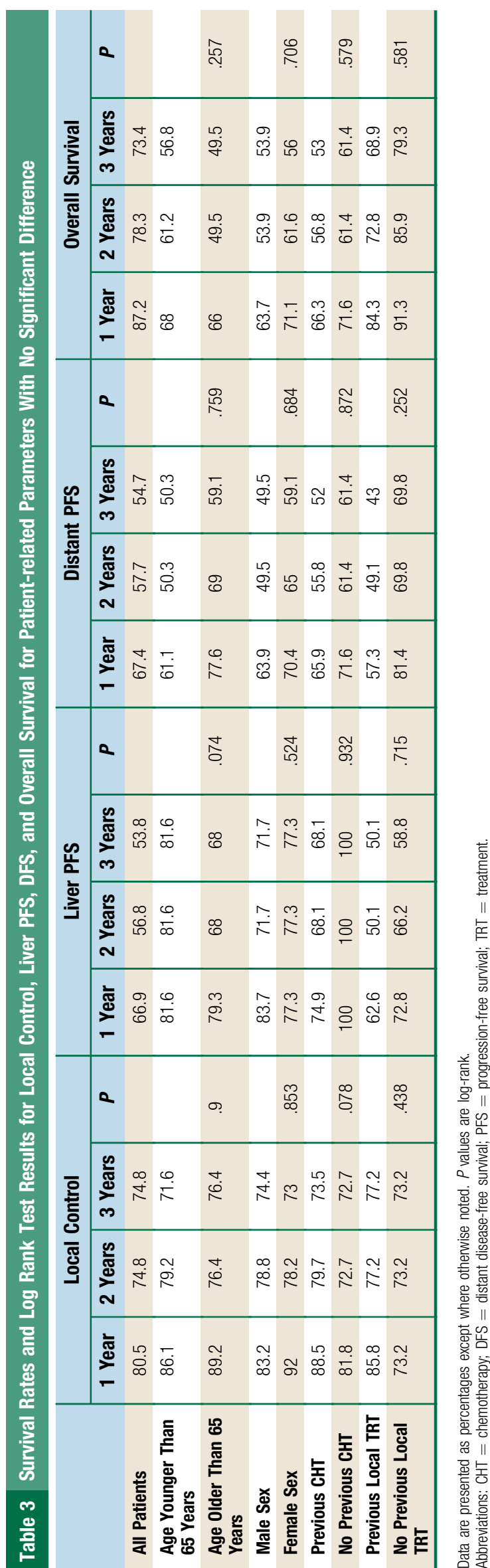

compare SBRT with other local treatment modalities including transcatheter arterial chemoembolization, radiofrequency ablation, or surgery. The patients were referred to and later followed in different institutions, so no standardized imaging protocols were used. Finally, longer patient follow-up would enable us to report more accurately on the interaction between delivered dose, LC, and OS.

\section{Conclusion}

We present data of 42 patients treated for 55 unresectable hepatic-only oligometastasis with controlled primary disease using the CyberKnife system. Our data are encouraging with outcome numbers at the higher end of the SBRT literature regarding LC and OS and comparable with the OS data for surgical and tumor ablation series. Most of our patients were treated in 3 fractions of 15 Gy with low toxicity rates. We propose a prescription threshold dose of $45 \mathrm{~Gy}$ on the CTV and $>43$ Gy on the PTV to at least $95 \%$ of both volumes to significantly improve LC. Our results support the potential paradigm shift whereby the use of SBRT in truly oligometastatic (ie, oligorecurrent) liver disease could benefit patients with liver metastases. However, longer follow-up is required especially concerning patient selection and fractionation schedules, to effectively determine the position of SBRT relative to surgical resection and other invasive techniques.

\section{Clinical Practice Points}

- Oligorecurrent liver metastases are frequently encountered in oncology and are associated with an unfavorable prognosis if untreated (3-year survival rate of approximately $3 \%$ ).

- Surgical resection as standard of care potentially increase the 10 -year OS up to $22 \%$ to $43 \%$, however, only one-quarter of the patients are eligible for such treatment.

- Other possible localized treatments are embolization, thermic ablation, and radiotherapy.

- A special form of radiotherapy (eg, SBRT) showed a potential increase of LC, but OS data are scarce.

- Using robotic SBRT with tumor tracking capacity (eg, the CyberKnife platform) might further improve clinical outcome.

- Clinical factors such performance status and histology influences LC, whereas age tends to affect liver-specific PFS.

- Using CyberKnife with real-time tumor tracking, biological treatment planning parameters (dose volume limits to target volumes) with certain minimum thresholds are crucial.

\section{Disclosure}

The authors report no conflicts of interest. The authors alone are responsible for the content and writing of the paper.

\section{Supplemental Data}

Supplemental figure accompanying this article can be found in the online version at http://dx.doi.org/10.1016/j.clcc.2017.03.006.

\section{References}

1. Yuan ZY, Meng MB, Liu CL, et al. Stereotactic body radiation therapy using the CyberKnife system for patients with liver metastases. Onco Targets Ther 2014; 7: 915-23.

2. Bengmark S, Hafström L. The natural history of primary and secondary malignant tumors of the liver. I. The prognosis for patients with hepatic metastases from colonic and rectal carcinoma by laparotomy. Cancer 1969; 23:198-202. 


\section{Patrick Berkovic et al}

3. Mohammad WM, Balaa FK. Surgical management of colorectal liver metastases. Clin Colon Rectal Surg 2009; 1:225-32.

4. Choti MA, Sitzmann JV, Tiburi MF, et al. Trends in long-term survival following liver resection for hepatic colorectal metastases. Ann Surg 2002; 235:759-66.

5. Wagner JS, Adson MA, Van Heerden JA, Adson MH, Ilstrup DM. The natura history of hepatic metastases from colorectal cancer. A comparison with resective treatment. Ann Surg 1984; 199:502-8.

6. Akgül Ö, Çetinkaya E, Ersöz Ş, Tez M. Role of surgery in colorectal cancer liver metastases. World J Gastroenterol 2014; 20:6113-22.

7. Van Cutsem E, Nordlinger B, Cervantes A, ESMO Guidelines Working Group. Advanced colorectal cancer: ESMO Clinical Practice Guidelines for treatment. Ann Oncol 2010; 21(suppl 5):v93-7.

8. Corbin KS, Hellman S, Weichselbaum RR. Extracranial oligometastases: a subset of metastases curable with stereotactic radiotherapy. J Clin Oncol 2013; 31:1384 90.

9. Goodman BD, Mannina EM, Althouse SK, Maluccio MA, Cárdenes HR. Longterm safety and efficacy of stereotactic body radiation therapy for hepatic oligometastases. Pract Radiat Oncol 2015; 6:86-95.

10. Lemke J, Cammerer G, Ganser J, et al. Survival and prognostic factors of colorectal liver metastases after surgical and nonsurgical treatment. Clin Colorectal Cancer 2016; 15:e183-92.

11. Stintzing S, Hoffmann RT, Heinemann V, Kufeld M, Muacevic A. Frameless single-session robotic radiosurgery of liver metastases in colorectal cancer patients. Eur J Cancer 2010; 46:1026-32.

12. Kennedy AS, Ball DS, Cohen SJ, et al. Safety and efficacy of radioembolization in elderly ( $>70$ years) and younger patients with unresectable liver-dominant colorectal cancer. Clin Colorectal Cancer 2016; 15:141-151e6.

13. Yoon SS, Tanabe KK. Surgical treatment and other regional treatments for colorectal cancer liver metastases. Oncologist 1999; 4:197-208.

14. Stintzing S, Grothe A, Hendrich S, et al. Percutaneous radiofrequency ablation (RFA) or robotic radiosurgery (RRS) for salvage treatment of colorectal liver metastases. Acta Oncol 2013; 52:971-7.

15. Ravikumar TS. Interstitial therapies for liver tumors. Surg Oncol Clin N Am 1996; 5:365-77.

16. Solbiati L, Livraghi T, Goldberg SN, et al. Percutaneous radio-frequency ablation of hepatic metastases from colorectal cancer: long-term results in 117 patients. Radiology 2001; 221:159-66.

17. Oshowo A, Gillams A, Harrison E, Lees WR, Taylor I. Comparison of resection and radiofrequency ablation for treatment of solitary colorectal liver metastases. $B r$ J Surg 2003; 90:1240-3.

18. Dawson LA. Overview: where does radiation therapy fit in the spectrum of liver cancer local-regional therapies? Semin Radiat Oncol 2011; 21:241-6.

19. Fiorentini G, Aliberti C, Tilli M, et al. Intra-arterial infusion of irinotecan-loaded drug-eluting beads (DEBIRI) versus intravenous therapy (FOLFIRI) for hepatic metastases from colorectal cancer: final results of a phase III study. Anticancer Res 2012; 32:1387-95.

20. Schefter TE, Kavanagh BD. Radiation therapy for liver metastases. Semin Radiat Oncol 2011;21:264-70.

21. Wulf J, Hädinger U, Oppitz U, Thiele W, Flentje M. Stereotactic boost irradiation for targets in the abdomen and pelvis. Radiother Oncol 2004; 70:31-6.
22. Høyer M, Swaminath A, Bydder S, et al. Radiotherapy for liver metastases: a review of evidence. Int I Radiat Oncol Biol Phys 2012; 82:1047-57.

23. Kim H, Gill B, Beriwal S, Huq MS, Roberts MS, Smith KJ. Cost effectiveness analysis of stereotactic body radiation therapy compared with radiofrequency ablation for inoperable colorectal liver metastases. Int J Radiat Oncol 2016; 95 : 1175-83.

24. Tree AC, Khoo VS, Eeles RA, et al. Stereotactic body radiotherapy for oligometastases. Lancet Oncol 2013; 14:e28-37.

25. Salama JK, Milano MT. Radical irradiation of extracranial oligometastases. J Clin Oncol 2014; 32:2902-12

26. Rusthoven KE, Kavanagh BD, Cardenes H, et al. Multi-institutional phase I/II trial of stereotactic body radiation therapy for liver metastases. J Clin Oncol 2009; 27:1572-8.

27. Boda-Heggemann J, Dinter D, Weiss C, et al. Hypofractionated image-guided breath-hold SABR (stereotactic ablative body radiotherapy) of liver metastases-clinical results. Radiat Oncol 2012; 7:92.

28. Niibe Y, Hayakawa K. Oligometastases and oligo-recurrence: the new era of cancer therapy. Jpn J Clin Oncol 2010; 40:107-11.

29. Timmerman RD. An overview of hypofractionation and introduction to this issue of seminars in radiation oncology. Semin Radiat Oncol 2008; 18:215-22.

30. Eisenhauer EA, Therasse P, Bogaerts J, et al. New Response Evaluation Criteria In Solid Tumours: revised RECIST guideline (version 1.1). Eur J Cancer 2009; 45: 228-47.

31. US Department of Health and Human Services. Common terminology criteria for adverse events (CTCAE) version 4.0. Bethesda, MD: National Cancer Institute. 2009 (09-5410).

32. McKinney W. Data structures for statistical computing in python. In: van de Voort S, Millman J, eds. Proceedings of the 9th Python in Science Conference, Vol. 445. Austin, TX: SciPy; 2010:51-6.

33. Ahmed KA, Caudell JJ, El-Haddad G, et al. Radiosensitivity differences between metastases based on primary histology suggest implications for clinical outcomes follwoing SBRT. Int I Radiat Oncol Biol Phys 2016; 95:1399-404.

34. Chang DT, Swaminath A, Kozak M, et al. Stereotactic body radiotherapy for colorectal liver metastases: a pooled analysis. Cancer 2011; 117:4060-9.

35. Takeda A, Sanuki N, Tsurugai Y, Oku Y, Aoki Y. Stereotactic body radiotherapy for patients with oligometastases from colorectal cancer: risk-Adapted dose prescription with a maximum dose of 83-100 Gy in five fractions. J Radiat Res 2016; 57:400-5.

36. Stintzing S, Hoffmann RT, Heinemann V, Kufeld M, Rentsch M, Muacevic A. Radiosurgery of liver tumors: value of robotic radiosurgical device to treat liver tumors. Ann Surg Oncol 2010; 17:2877-83.

37. Sanabria J, Goyal K, Einstein D, et al. Cyberknife stereotactic body radiation therapy for nonresectable tumors of the liver: preliminary results. HPB Surg 2010; 2010:309780.

38. Swaminath A, Massey C, Brierley JD, et al. Accumulated delivered dose response of stereotactic body radiation therapy for liver metastases. Int I Radiat Oncol Biol Phys 2015; 93:639-48.

39. Méndez Romero A, Wunderink W, Hussain SM, et al. Stereotactic body radiation therapy for primary and metastatic liver tumors: a single institution phase I-II study. Acta Oncol 2006; 45:831-7. 


\section{SBRT for Unresectable Hepatic Oligorecurrence}

\section{Supplemental Figure 1 Kaplan-Meier Curves and Log-Rank Test for Local Control, Liver Progression-free Survival (PFS), Disease-free Survival, and Overall Survival for Patient-related Parameters With No Statistically Significant Effect}
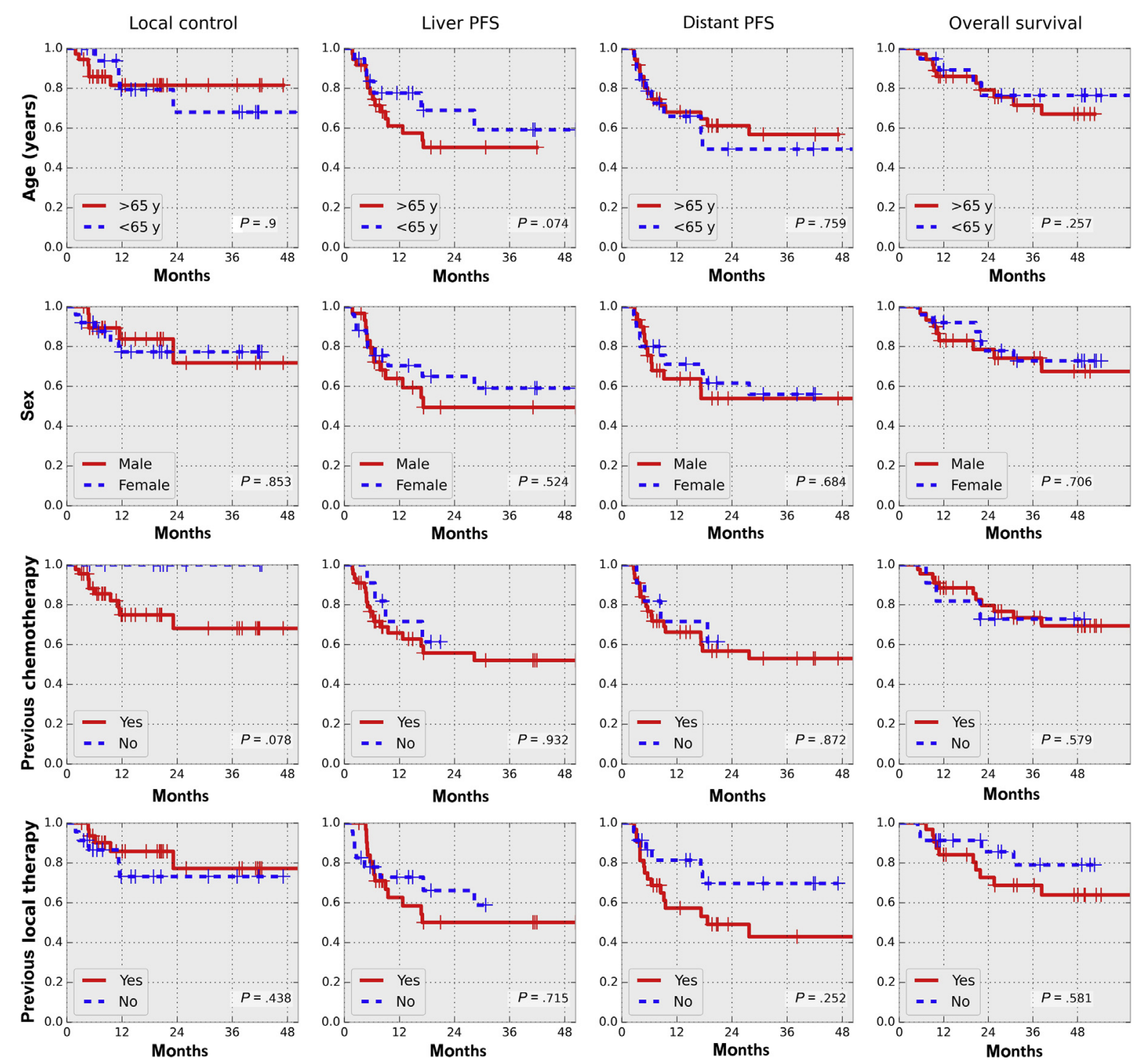